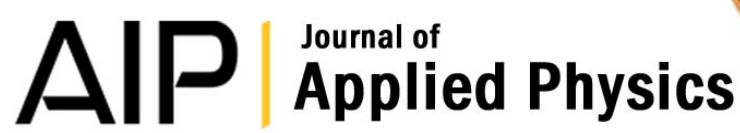

Tuning the emission colour by manipulating terbium-terbium interactions: Terbium doped aluminum nitride as an example system

Felix Benz, Horst P. Strunk, Jakob Schaab, Ulrike Künecke, and Peter Wellmann

Citation: J. Appl. Phys. 114, 073518 (2013); doi: 10.1063/1.4818815

View online: http://dx.doi.org/10.1063/1.4818815

View Table of Contents: http://jap.aip.org/resource/1/JAPIAU/v114/i7

Published by the AIP Publishing LLC.

Additional information on J. Appl. Phys.

Journal Homepage: http://jap.aip.org/

Journal Information: http://jap.aip.org/about/about_the_journal

Top downloads: http://jap.aip.org/features/most_downloaded

Information for Authors: http://jap.aip.org/authors

\section{ADVERTISEMENT}

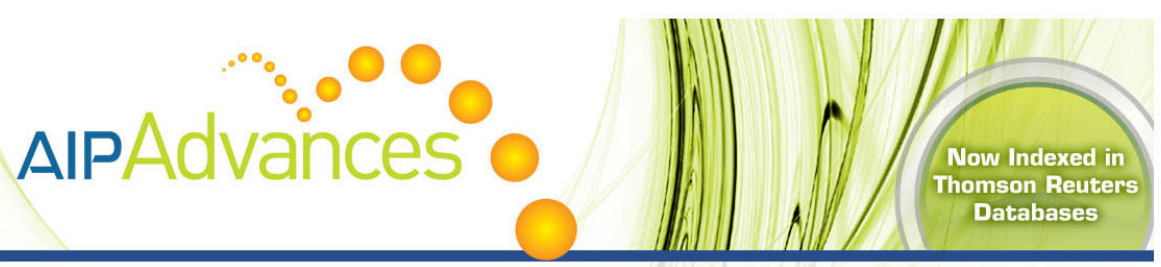

\section{Explore AIP's open access journal: Rapid publication \\ - Article-level metrics \\ - Post-publication rating and commenting}




\title{
Tuning the emission colour by manipulating terbium-terbium interactions: Terbium doped aluminum nitride as an example system
}

\author{
Felix Benz, ${ }^{1, a), b)}$ Horst P. Strunk, ${ }^{1}$ Jakob Schaab, ${ }^{1, c)}$ Ulrike Künecke, ${ }^{2}$ and Peter Wellmann ${ }^{2}$ \\ ${ }^{1}$ Institute of Materials Science, University of Stuttgart, Heisenbergstr. 3, 70569 Stuttgart, Germany \\ ${ }^{2}$ Department of Materials Science-Materials for Electronics and Energy Technology, \\ University of Erlangen-Nürnberg, Martensstr. 7, 91058 Erlangen, Germany
}

(Received 7 June 2013; accepted 2 August 2013; published online 21 August 2013)

\begin{abstract}
Terbium-terbium interactions in terbium doped semiconductors and insulators may lead to the so-called cross-relaxation process, which increases the ${ }^{5} \mathrm{D}_{4}$ (green) emission of the terbium ions at the cost of the ${ }^{5} \mathrm{D}_{3}$ (blue) luminescence intensity. This effect can generally be reduced by increasing the distance between an excited ion and the nearest ion in the ground state. A straightforward measure is to use a specimen with a decreased terbium concentration. The alternative is to increase the intensity of the excitation (either by photons or electrons) and thereby to reduce the population of terbium ions in the ground state. This paper works this process out with the example of AlN:Tb on the basis of a model and respective experimental results. As will be seen, stronger excitation causes in essence more $\mathrm{Tb}$ ions to be excited, thus less ions in the ground state which increases the distance between an excited and the nearest ground state ions. This hinders energy transfer between the terbium ions and thus counteracts the cross-relaxation process. The advantage of changing the excitation intensity lies in the possibility to deliberately shift the apparent colour of the Tb luminescence from a single specimen between green and blue. (C) 2013 AIP Publishing LLC. [http://dx.doi.org/10.1063/1.4818815]
\end{abstract}

\section{INTRODUCTION}

Rare earth doped semiconductors ${ }^{1}$ exhibit narrow emission lines, which are almost insensitive to external factors like the temperature ${ }^{2}$ or the surrounding matrix. ${ }^{3}$ This insensitivity and the narrow spectral width of the emission lines makes these materials promising candidates for phosphors, ${ }^{1,4}$ optical data transfer, ${ }^{5}$ and low cost bio sensing. ${ }^{6}$ In fact, europium was and still is widely used particularly for red emission in cathode ray tube (CRT) displays, electric discharge lightning and in plasma screens. ${ }^{7}$ However, the radiative lifetimes of the rare earth ions are, compared to other atomic processes, quite long (typically on the order of milliseconds) ${ }^{8-10}$ Therefore, non-radiative processes, e.g., resulting from ion interactions, emerge as undesired intensity reducing competitors.

An important non-radiative process is, for example, a transfer of energy between individual ions which is the higher in probability the smaller the interspace is. Repetition of this transfer enhances the chance to lose the excitation energy to a non-radiative recombination process and thus to a corresponding reduction in the luminescence intensity. ${ }^{11}$ This energy transfer is practically independent of the structure and chemical nature of the matrix material, as long as the bandgap is sufficiently large. Two mechanisms can be discriminated: first, "concentration quenching" by which the luminescence intensity of the whole spectrum is reduced by a smaller average interspace (higher concentration) of the

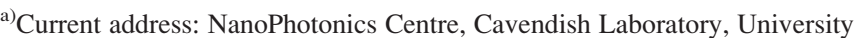
of Cambridge, Cambridge CB3 OHE, United Kingdom.

b)Electronic mail: felix.benz@imw.uni-stuttgart.de.

${ }^{c}$ Current address: ETH Zürich, Department of Materials (D-MATL), Multifunctional Ferroic Materials, Wolfgang Pauli Str. 10, CH-8093 Zürich, Switzerland.
}

rare earth ions. The second mechanism, "cross relaxation," is very pronounced in the case of terbium. It is sketched in Figure 1. One ion, $A$, is excited into the ${ }^{5} \mathrm{D}_{3}$ state, from which state a part of the excitation energy, by relaxing into the ${ }^{5} \mathrm{D}_{4}$ state, is transferred to the nearby ion, B, exciting it into the state ${ }^{7} \mathrm{~F}_{0}$. This process occurs practically in resonance, since the involved energy amounts in both ions are

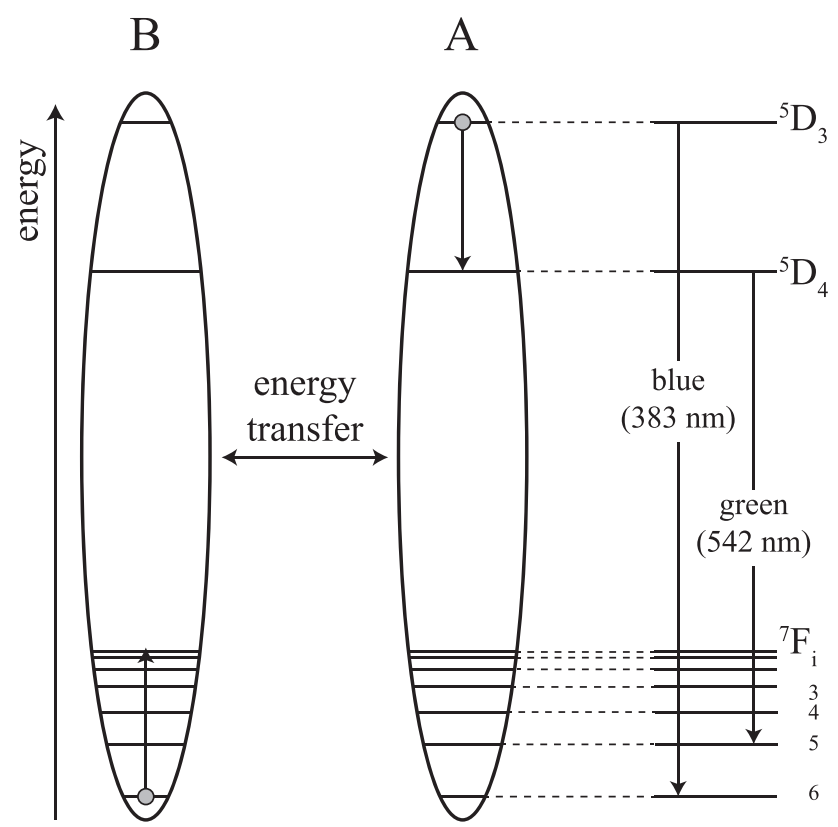

FIG. 1. Schematic illustration of the cross-relaxation process: Ion A, excited into the ${ }^{5} \mathrm{D}_{3}$ state transfers a part of its excitation energy to ion $B$ nearby. Thereby the ion A relaxes into the ${ }^{5} \mathrm{D}_{4}$ state and ion $\mathrm{B}$ is excited into the ${ }^{7} \mathrm{~F}_{0}$ state. The process suppresses effectively the emission from ${ }^{5} \mathrm{D}_{3}$ ("blue emission") and facilitates the emission from ${ }^{5} \mathrm{D}_{4}$ ("green emission"). 
nearly equal. It is obvious that in this case (Figure 1, transition arrows at right) the luminescence intensity quenching is energy/wavelength selective: the blue ${ }^{5} \mathrm{D}_{3}$ emission of the terbium ions is reduced to the advantage of the green ${ }^{5} \mathrm{D}_{4}$ emission. (The state ${ }^{7} \mathrm{~F}_{0}$ very probably relaxes by phonon emission.)

Cross-relaxation can thus be reduced and suppressed by choosing an appropriately small rare earth concentration (correspondingly large ion interspace) as has recently been proven by a significant increase of the blue emission relative to the green emission from terbium-doped lead phosphate glasses ${ }^{12}$ and in terbium-doped yttrium oxyorthosilicates. ${ }^{13}$ Blue emission can also be enhanced by a high excitation intensity ${ }^{14}$ that causes an excessive population of excited states which in turn increases the distance between an excited ion and a still available ion in the ground state. The present paper treats jointly both the dependency of the terbium luminescence on rare earth concentration and on excitation intensity.

\section{THEORETICAL ASPECTS}

Two common atomistic models exist for interactions leading to energy transfer and thereby causing both crossrelaxation and concentration quenching: exchange interaction that requires the wavefunctions to overlap and electrostatic interaction that acts over much larger distances. In the case of exchange interaction, the probability for an energy transfer $p_{\text {exch. }}^{E T}$ can be expressed by ${ }^{15}$

$$
p_{\text {exch. }}^{E T} \propto \exp \left(-\frac{r}{r_{0}}\right)
$$

with the distance between the two ions, $r$, and a characteristic atomic length, $r_{0}$. In the case of electrostatic interaction, the Förster formula gives the probability for an energy transfer $p_{\text {elec. }}^{E T} 16$

$$
p_{\text {elec. }}^{E T} \propto\left(\frac{r}{r_{F}}\right)^{-m}
$$

with $m$ depending on the mode of interaction $(m=6,8,10$ for dipole-dipole, dipole-quadrupole, and quadrupolequadrupole interaction, respectively) and the Förster radius, $r_{F}$. For real materials, a combination of both processes is expected. The case of exchange interaction shall be outlined here as an example.

Equations (1) and (2) give the energy transfer probabilities for a pair of ions only. In order to describe an ensemble of many ions, it is feasible to replace the distance between the ions, $r$, by an average ion interspace, $\langle r\rangle$. This interspace can be calculated from the ion number density, $n$, e.g., by the distribution function $\rho(r)$ proposed by Chandrasekhar ${ }^{17}$ for a random distribution modified ${ }^{11}$ by a minimum distance $d_{0}$ that is given by twice the ionic diameter

$$
\rho(r)=\left\{\begin{array}{l}
0 \text { if } \quad r<d_{0} \\
\left(r^{2}-d_{0}^{2}\right) n \times \exp \left[-\frac{4}{3} \pi n\left(r^{3}-d_{0}{ }^{3}\right)\right] \text { if } \quad r \geq d_{0},
\end{array}\right.
$$

$$
\langle r\rangle=\frac{\int_{0}^{\infty} \mathrm{d} r \rho(r) \times r}{\int_{0}^{\infty} \mathrm{d} r \rho(r)} .
$$

In view of the cross-relaxation, however, the interspace between an ion in the excited state and an ion in the ground state $\left\langle r^{e x-g r}\right\rangle$ is of interest instead of the overall ion interspace as calculated from Eqs. (3) and (4). Figure 2 illustrates this fact: For only few excited ions, the overall ion interspace $\langle r\rangle$ is approximately equal to the distance between an excited ion and the nearest ion in the ground state $\left\langle r^{e x-g r}\right\rangle$ (Figure 2(a)). For larger number densities of excited ions (cf. Figure 2(b)) $\left\langle r^{e x-g r}\right\rangle$ is always larger than $\langle r\rangle$.

Since the overall number density is constant $n=n_{g r}+n_{\text {exc }}$ (with the number density of unexcited $n_{g r}$ and excited ions $n_{\text {exc }}$ ), both distances depend on each other. The interspace is generally proportional to $n^{-\frac{1}{3}}$, the proportionality factor $d$ accounts for the used distribution function. Therefore, the following equations yield for the interspace $r^{e x-g r}$ :

$$
\begin{gathered}
\left\langle r^{e x-g r}\right\rangle=\frac{d}{\left(n_{g r}\right)^{\frac{1}{3}}}=\frac{d}{(n)^{\frac{1}{3}}} \times\left(\frac{n}{n-n_{e x c}}\right)^{\frac{1}{3}}, \\
\frac{\left\langle r^{e x-g r}\right\rangle}{\langle r\rangle}=\left(\frac{1}{1-x_{e x c}}\right)^{\frac{1}{3}},
\end{gathered}
$$

with the fraction of ions in the excited state $x_{\text {exc }}=\frac{n_{e x c}}{n}$. Equation (6) is plotted in Fig. 3 as a continuous line.

We have used our statistical model, previously developed for describing concentration quenching, ${ }^{18}$ to test this equation. A certain number of points (17500; 15000; $12500 ; 10000$ and 1000) randomly distributed in a cube of fixed edge length has been calculated with this Monte-Carlo (a)
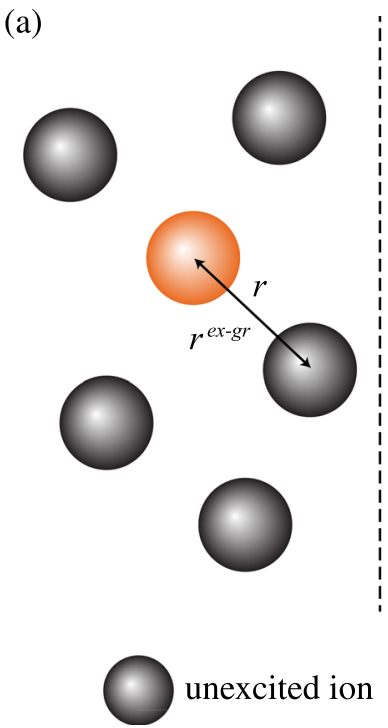

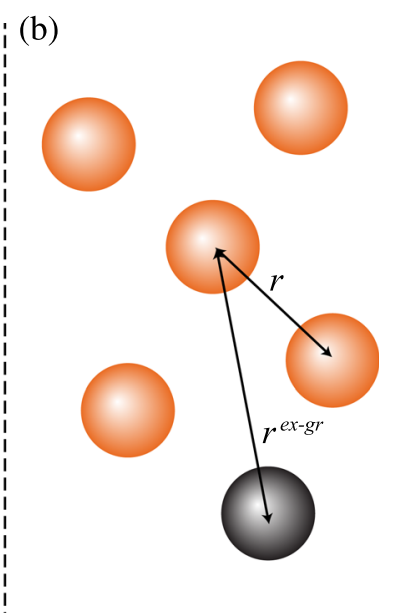

excited ion
FIG. 2. Nearest neighbour distance $r$ and distance between one excited ion and the nearest ion in the ground state $r^{e x-g r}$ for both only few excited ions (a) or a larger number of excited ions (b). 


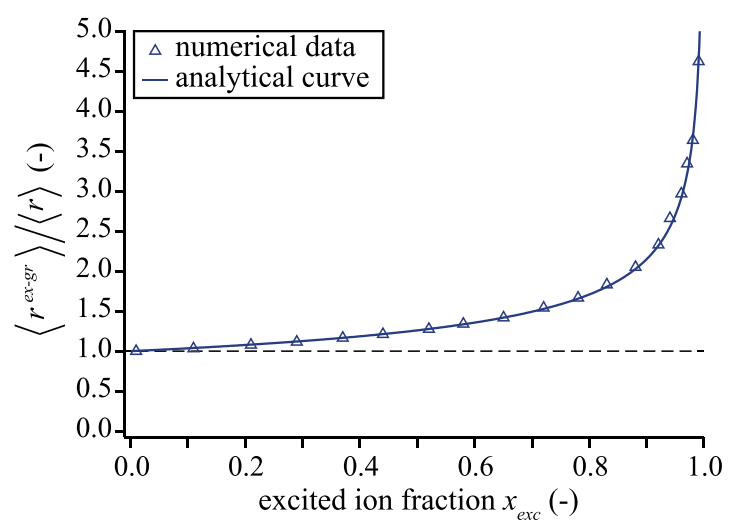

FIG. 3. Average excited to unexcited ion interspace $\left\langle r^{e x-g r}\right\rangle$ normalised with the interspace of all ions $\langle r\rangle$. Numerically determined data are shown together with the analytical function shown in Eq. (6).

based simulation. Subsequently, a certain fraction of these points were assumed to be "excited." A numerical algorithm calculated $\langle r\rangle$ and $\left\langle r^{e x-g r}\right\rangle$. Figure 3 contains the numerically obtained data. The data are identical for all number densities, i.e., analytical and statistical descriptions agree perfectly.

For determining an equation for the ${ }^{5} \mathrm{D}_{3} /{ }^{5} \mathrm{D}_{4}$ intensity ratio, which can experimentally be checked, we use a rate equation model ${ }^{11}$ with three states (ground state, excited ${ }^{5} \mathrm{D}_{3}$ and ${ }^{5} \mathrm{D}_{4}$ states) and assume that the ions are excited in the ${ }^{5} \mathrm{D}_{3}$ state only (see Appendix). The used model yields the following equation:

$$
\frac{I\left({ }^{5} \mathrm{D}_{3}\right)}{I\left({ }^{5} \mathrm{D}_{4}\right)} \propto \frac{1}{a+b \times p^{E T}} .
$$

For the sake of simplicity, we set $a=b=1$ for the following discussion, therefore, we obtain $I\left({ }^{5} \mathrm{D}_{3}\right) / I\left({ }^{5} \mathrm{D}_{4}\right) \rightarrow 1$ for $p^{\mathrm{ET}} \rightarrow 0$ and $I\left({ }^{5} \mathrm{D}_{3}\right) / I\left({ }^{5} \mathrm{D}_{4}\right) \rightarrow 0$ for $p^{\mathrm{ET}} \rightarrow \infty$.

Using this equation together with Eqs. (1) and (6), one can obtain a map for the ${ }^{5} \mathrm{D}_{3} /{ }^{5} \mathrm{D}_{4}$ intensity ratios as a function of both concentration and excitation intensity. Figure 4 shows this map for arbitrary values of both parameters.

\section{EXPERIMENTAL ASPECTS}

Eight aluminum nitride samples with different terbium number densities between approximately $1.0 \times 10^{21} \mathrm{~cm}^{-3}$

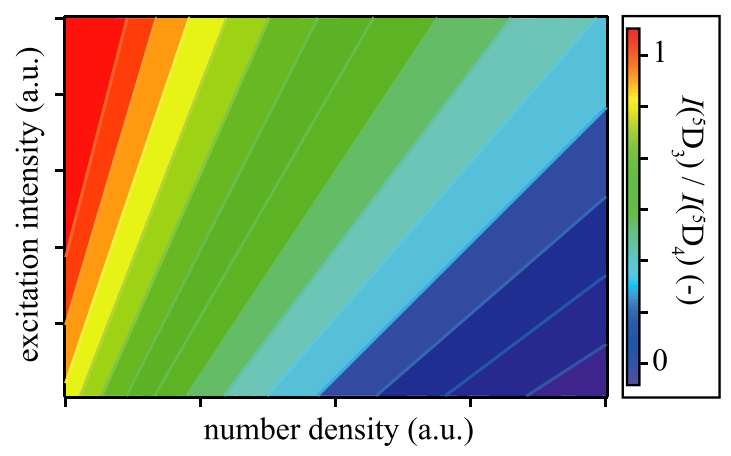

FIG. 4. $\frac{I\left({ }^{5} \mathrm{D}_{3}\right)}{I\left({ }^{5} \mathrm{D}_{4}\right)}$ intensity ratio as a function of both ion number density and excitation intensity. The number density is used for better comparison with experimental results. Shown are isolines with arbitrary values, in order to demonstrate that all isolines are straight. and $1.6 \times 10^{21} \mathrm{~cm}^{-3}$ were prepared by reactive DC magnetron sputtering. Two aluminum targets $(150 \mathrm{~W}$ sputtering power each) and one terbium target (sputtering power variable between 2 and $12 \mathrm{~W}$ ) were used for adjusting the terbium concentration. The sputtering atmosphere consisted of a mixture of argon and nitrogen with a total pressure of $6 \times 10^{-3}$ mbar. After sputtering, the samples were annealed at $1000\left({ }^{\circ} \mathrm{C}\right.$ for $30 \mathrm{~min}$ under a 1 bar nitrogen atmosphere.

The terbium number density was determined by inductively coupled plasma optical emission spectroscopy (ICPOES) of samples with a known volume. Supplemental X-ray photoelectron spectroscopy investigations matched with the results obtained by ICP-OES. The microstructure of the samples consists basically of a columnar grain structure (determined by transmission electron microscopy, not shown here, see Ref. [11]). X-ray diffraction confirmed the aluminum nitride wurtzite structure.

Photoluminescence spectroscopy (PL) was applied to measure the emission lines originating from the transitions ${ }^{5} \mathrm{D}_{3} \rightarrow{ }^{7} \mathrm{~F}_{6}$ and ${ }^{5} \mathrm{D}_{4} \rightarrow{ }^{7} \mathrm{~F}_{6,5}$. A Fluorolog 3 FL-22 spectrometer (Horiba Scientific), equipped with a standard photomultiplier tube served to collect the data. The spectra were recorded with a sampling of 1 point per nanometre (emission bandpass: $4 \mathrm{~nm}$ ). The integration time per point was $2 \mathrm{~s}$, each spectrum was recorded five times and averaged. The acquired spectra were baseline corrected and integrated in order to obtain the respective integrated intensities. As excitation source $304 \mathrm{~nm}$ radiation as monochromised from a $450 \mathrm{~W}$ xenon lamp was used, if not otherwise stated. This particular wavelength was used since it corresponds to the largest intensity obtained from a photoluminescence excitation (PLE) spectrum as shown in Figure 5(a) by the solid line. There is a second peak at $255 \mathrm{~nm}$ which actually corresponds to an even higher excitation efficiency. This becomes obvious (see below) when the PLE intensities are normalised to the lamp spectrum, see solid line in Figure 5(b).

The integrated excitation intensity as monitored by a standard Si photodiode, previously calibrated with appropriate optical sensors (Coherent LM-2 UV and LM-2 VIS), was varied by changing the bandpass of the excitation monochromator. Figure 5(c) shows the light power densities for the used bandpasses at a central wavelength of $304 \mathrm{~nm}$.

At this point, we would like to comment in some detail on the results shown in Figure 5 as they lead to a first notion of the molecular processes during annealing. The PLE peak around $304 \mathrm{~nm}$ (cf. Figure 5(a)) is present in all terbium doped samples be it in the as-grown or annealed states. Undoped aluminum nitride samples exhibit an emission peak at around $304 \mathrm{~nm} .{ }^{19}$ As-grown terbium doped samples also exhibit this emission peak (cf. Figure 5(b)) which, however, disappears on annealing. These results indicate that (i) the excitation is due to excitation of an intrinsic lattice defect of AlN, (ii) in the presence of terbium a high energy transfer probability from the excited lattice defect to the terbium ion exists, (iii) which causes the $304 \mathrm{~nm}$ emission to decrease during annealing treatments although it is still present in the PLE spectrum. These results suggest that this (otherwise unidentified) lattice defect resides in intimate vicinity to a terbium ion. In contrast, no luminescence corresponding to the 
(a)

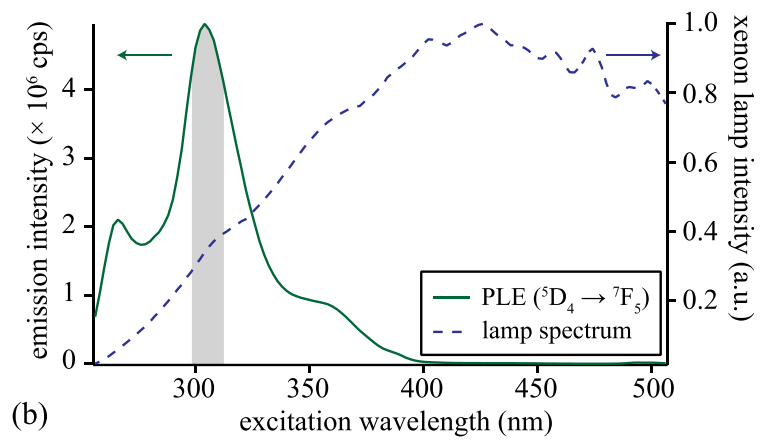

(b)

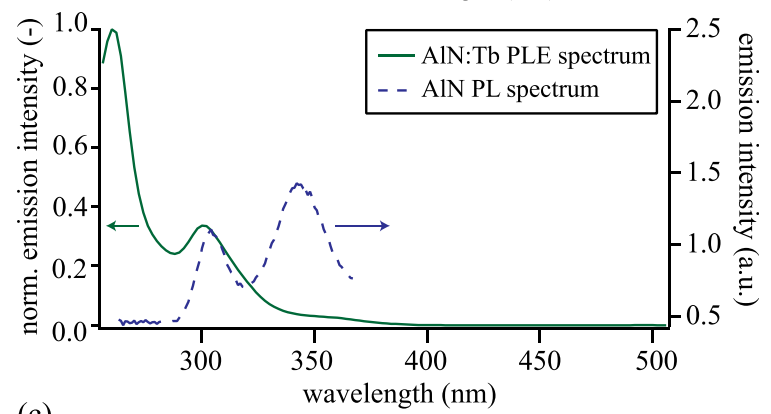

(c)

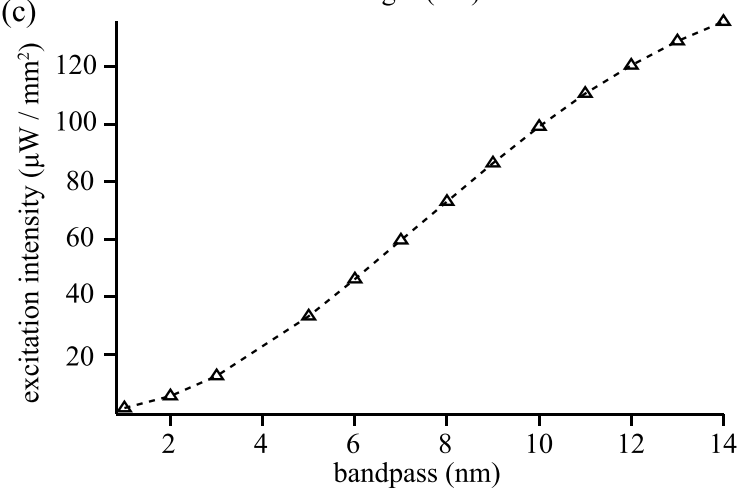

FIG. 5. (a) Unprocessed photoluminescence excitation (PLE) spectrum together with the lamp spectrum of the used xenon lamp. The area shaded grey in (a) indicates the approximate spectral region for excitation with a $14 \mathrm{~nm}$ bandpass. (b) Normalised PLE spectrum (solid line) together compared to the emission spectrum of an as-grown AlN:Tb sample, dashed line. (Please note: This matrix related emission has also been observed for pure AlN deposited by the same method. The emission vanishes if the doped samples are annealed, the undoped samples show the same luminescence in the as-grown and annealed state.) (c) Light power densities for the used bandpasses.

second peak at approximately $255 \mathrm{~nm}$ was observed. This peak thus might correspond to a charge transfer excitation of the terbium ions as predicted by Dorenbos et al. for an energy of $\approx 5 \mathrm{eV}(=248 \mathrm{~nm}){ }^{20}$

\section{RESULTS AND DISCUSSION}

Figure 6 shows a compilation of experimental spectra for different light power densities as obtained from the sample with $1.0 \times 10^{21} \mathrm{~cm}^{-3}$. All spectra are normalised to the highest observed intensity. One can clearly see that the peak corresponding to the ${ }^{5} \mathrm{D}_{3} \rightarrow{ }^{7} \mathrm{~F}_{6}$ transition increases relative to the ${ }^{5} \mathrm{D}_{4} \rightarrow{ }^{7} \mathrm{~F}_{6}$ peak with increasing excitation density. For comparison also shown are cathodoluminescence data (dotted curve) obtained by Lozykowski et al. ${ }^{21}$ with very high beam currents. This spectrum demonstrates that for

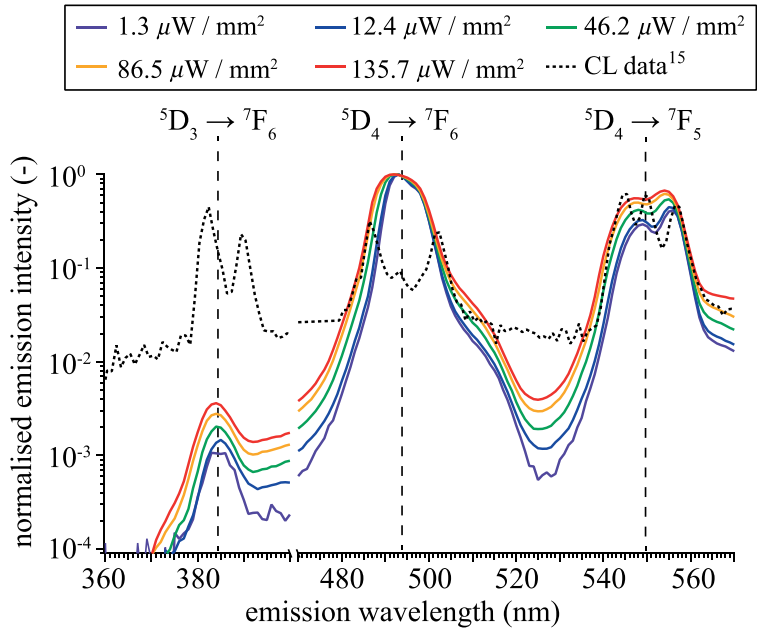

FIG. 6. Spectra recorded with different light power densities from the same spot on one sample (AlN:Tb with $1.0 \times 10^{21} \mathrm{~cm}^{-3}$ ). The dotted spectrum is from a $\mathrm{GaN}: \mathrm{Tb}$ sample investigated by cathodoluminescence with a beam current of $75 \mu \mathrm{A}$ and an acceleration voltage of $5 \mathrm{kV},{ }^{21}$ for a further explanation see text.

sufficiently high excitation intensity a ${ }^{5} \mathrm{D}_{3} /{ }^{5} \mathrm{D}_{4}$ ratio of almost unity is possible.

Figure 7 shows the experimentally determined ${ }^{5} \mathrm{D}_{3} /{ }^{5} \mathrm{D}_{4}$ ratios as a function of the terbium number density and the excitation intensity. The proposed trend, i.e., higher intensity from ${ }^{5} \mathrm{D}_{3}$ at lower concentrations and higher excitation intensities (cf. Figures 4 and 7) can clearly be seen. Furthermore, signal fluctuations cause some troughs that deviate from the anticipated linear isolines.

Some luminescence results obtained with a rather high excitation intensity are available from the work of Lozykowski et al. ${ }^{21}$ and are introduced in Figure 6. As obvious, a ${ }^{5} \mathrm{D}_{3} /{ }^{5} \mathrm{D}_{4}$ ratio of almost unity can be reached. However, directly comparing both excitation methods is difficult because of the completely different excitation processes: In the case of photoluminescence, the used radiation has an energy smaller than the bandgap and thus excitation of terbium ions occurs via direct excitation and/or localised bandgap states (like bound excitons, ${ }^{2}$ charge transfer states $^{20}$ or point defects). In this case, every photon can excite one of these states and thus one single ion at most. In the case of cathodoluminescence, each electron having a

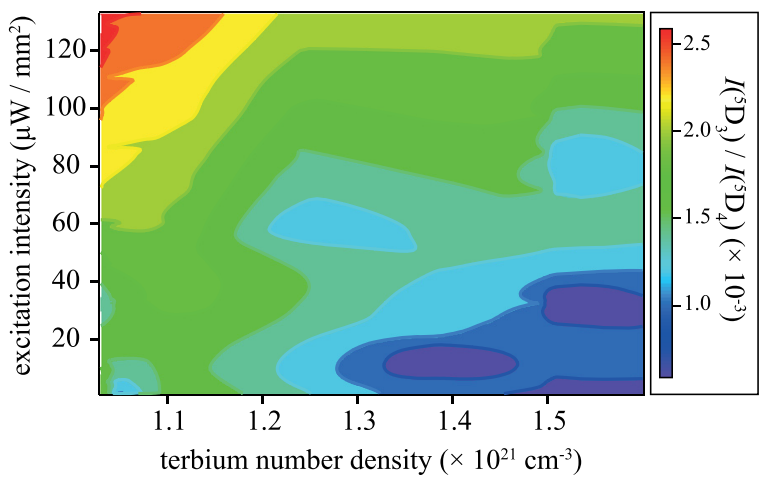

FIG. 7. Experimentally determined ${ }^{5} \mathrm{D}_{3} /{ }^{5} \mathrm{D}_{4}$ ratio for sputter deposited terbium doped aluminum nitride as a function of the terbium number density and the excitation intensity. 


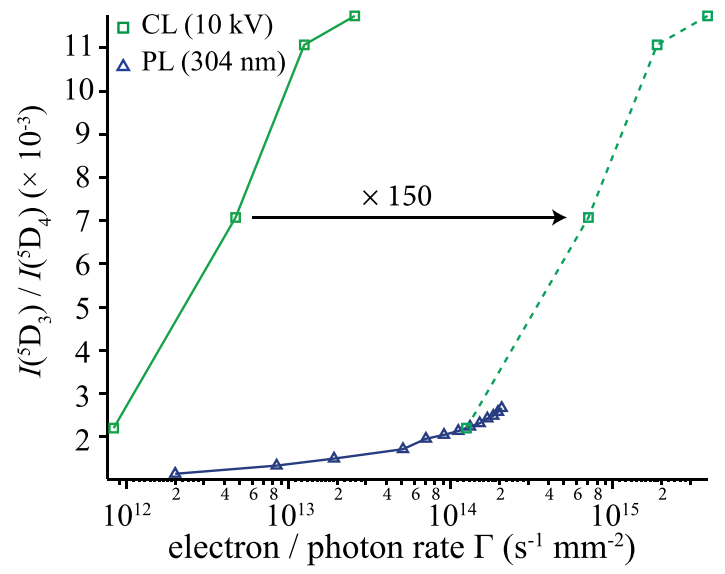

FIG. 8. Excitation efficiencies for electrons and photons. ${ }^{5} \mathrm{D}_{3} /{ }^{5} \mathrm{D}_{4}$ ratio: Green curves: CL with $10 \mathrm{keV}$ electron excitation, blue curve: PL with $304 \mathrm{~nm}$ photon excitation. The lines are guides to the eye. The terbium number density is approximately $1 \times 10^{21} \mathrm{~cm}^{-3}$. The CL curve shifted by a factor of 150 along the abscissa (see green dashed curve) suggests the excitation efficiency of one $10 \mathrm{keV}$ electron to be 150 times that of one $304 \mathrm{~nm}$ photon.

kinetic energy much larger than the energy required to produce one electron-hole pair can generate many electron-hole pairs, ${ }^{22}$ i.e., can excite many terbium ions at once. The excitation intensity $p_{\text {exc }}$ (that ultimately rules the population in the excited state) thus depends on two terms: the rate $\Gamma$ with which photons or electrons are impinging on the sample and an excitation efficiency $\eta(E)$ characteristic for the respective excitation mechanism

$$
p_{e x c}=\eta(E) \times \Gamma .
$$

This efficiency may thus be much higher for cathodoluminescence (CL) than for PL as seen in Figure 8. It shows for comparison reasons our experimental curve for excitation with electrons (beam current densities between $0.13 \frac{\mu \mathrm{A}}{\mathrm{mm}^{2}}$ and $4.10 \frac{\mu \mathrm{A}}{\mathrm{mm}^{2}}$ at an acceleration voltage of $10 \mathrm{kV}$ ) in addition to the photonic excitation curve. The slope of the CL curve is in fact rather steep (mind the logarithmic scale) indicating that the value of $\eta$ is considerably larger than for PL. One may associate this steep curve with the high excitation range of Figure 3. In fact, shifting the experimental CL curve to the right until it meets the photon curve indicates that one $10 \mathrm{keV}$ electron on average excites the same number of terbium ions than 150 photons. For comparison according to Owens and Peacock, ${ }^{22}$ the average energy for creating an electron-hole pair by one electron in aluminum nitride is $15.2 \mathrm{eV}$ (practically independent of the initial electron energy, see Klein ${ }^{23}$ ). Accordingly, one $10 \mathrm{keV}$ electron, as used here, would create 660 electron-hole pairs. Thus, it could be concluded that 100 electron-hole pairs have the same excitation probability as 23 photons with a wavelength of $304 \mathrm{~nm}$.

\section{CONCLUSION}

At sufficiently high excitation energy both blue $\left({ }^{5} \mathrm{D}_{3}\right)$ and green $\left({ }^{5} \mathrm{D}_{4}\right)$ luminescent emission of terbium can be excited. Earlier and recent work indicates that the intensity ratio of blue to green depends on the concentration of the ions, which was recently confirmed for terbium in glass and crystalline phosphor matrices. ${ }^{12,13}$ This intensity ratio depends also on the excitation intensity as already indicated earlier. ${ }^{14}$ Both dependencies on concentration and on excitation intensity have been treated and explained here in some detail on the basis of a rate equation model and with our new results complemented with literature results. The main outcome is that it is essentially the distance between an excited terbium ion and the nearest ion in the ground state (pivotal distance) which governs the ${ }^{5} \mathrm{D}_{3} /{ }^{5} \mathrm{D}_{4}$ luminescence ratio. The smaller this distance the higher the chance for cross relaxation, i.e., depopulation of the ${ }^{5} \mathrm{D}_{3}$ state (blue) in favour of the ${ }^{5} \mathrm{D}_{4}$ state (green) by transferring the corresponding energy difference with higher chance to the nearby ground state ion (exciting it within the ${ }^{7} \mathrm{~F}$ level family). This pivotal distance can simply be controlled by selecting an adequate terbium concentration, yielding a fixed apparent colour of the luminescence determined by the actual value of the green to blue emission ratio. This paper shows that increasing excitation intensity leads to an increasing number density of excited terbium ions, thus a lower density of ground state ions and a larger pivotal distance. Changing the excitation density controls the apparent colour of the luminescence from terbium between blue (high excitation intensity) to green (low excitation intensity) with one sample. The comparison of cathodoluminescence (high excitation range) with $30 \mathrm{keV}$ electrons to photoluminescence (low excitation range) with wavelength $304 \mathrm{~nm}$ suggests that 100 electronhole pairs produced by electrons pairs have approximately the same excitation probability as 23 photons.

\section{ACKNOWLEDGMENTS}

The authors acknowledge the support of the Thin Film Laboratory of the Max Planck Institute for Intelligent Systems, Stuttgart, Germany. A particular thank you goes to R. Völker and G. Richter. F.B. thanks W. M. Jadwisienczak (Ohio University) for making some of his CL data available to us.

\section{APPENDIX: RATE EQUATION MODEL}

The used rate equation model contains three states, namely the two excited states ${ }^{5} \mathrm{D}_{3}$ and ${ }^{5} \mathrm{D}_{4}$ and one ground state. The following equations describe the time evolution of the populations in the respective states:

$$
\begin{aligned}
\frac{\mathrm{d}}{\mathrm{d} t} n_{g r}= & -p_{\text {exc }} \times n_{g r}+\left(p_{\text {rad }}^{5 \mathrm{D}_{3}}+p_{\text {non-rad }}^{5 \mathrm{D}_{3}}\right) \times n^{5} \mathrm{D}_{3} \\
& +\left(p_{\text {rad }}^{5 \mathrm{D}_{4}}+p_{\text {non-rad }}^{5 \mathrm{D}_{4}}\right) \times n^{5} \mathrm{D}_{4}, \\
\frac{\mathrm{d}}{\mathrm{d} t} n_{5}{ }_{\mathrm{D}_{3}}= & +p_{\text {exc }} \times n_{g r}-\left(p_{\text {non-rad }}^{5}+p_{\text {rad }} \mathrm{D}_{3} \mathrm{D}_{3}\right) \times n^{n_{5} \mathrm{D}_{3}} \\
& -c \times p^{E T} \times n^{{ }_{5} \mathrm{D}_{3}}, \\
\frac{\mathrm{d}}{\mathrm{d} t} n_{5} \mathrm{D}_{4}= & -\left(p_{\text {rad }}^{5}+p_{\text {non-rad }}^{5}\right) \times n^{5} \mathrm{D}_{\mathrm{D}_{4}} \\
& +c \times p^{E T} \times n^{5} \mathrm{D}_{3},
\end{aligned}
$$


with the probabilities for excitation, radiative- and nonradiative recombination, and energy transfer $p_{\text {exc }}$, $p_{\text {rad }}^{i}, p_{\text {non-rad }}^{i}$, and $p^{E T}$, respectively. $n_{i}$ are the number densities in the respective states $i$. The constant $c$ (which has a value between 0 and 1) accounts for the fact that only a fraction of all energy transfers lead to cross-relaxation. We solved this equation for the stationary case $(t \rightarrow \infty)$ with the boundary conditions $n_{5} \mathrm{D}_{3}(0)=n_{5} \mathrm{D}_{4}(0)=0$ and $n_{g r}(0)=1$. The intensity ratio between ${ }^{5} \mathrm{D}_{3}$ and ${ }^{5} \mathrm{D}_{4}$ is then given by

$$
\frac{I\left({ }^{5} \mathrm{D}_{3}\right)}{I\left({ }^{5} \mathrm{D}_{4}\right)}=\frac{p_{\text {rad }}^{5} \times{ }^{\mathrm{D}_{3}} \mathrm{D}_{3}(\infty)}{p_{\text {rad }}^{5} \times{ }^{n_{4}} \mathrm{D}_{4}(\infty)} .
$$

We used the solutions of Eqs. (A2) and (A3) for calculating the steady state populations $n_{5} \mathrm{D}_{3}(\infty)$ and $n_{5} \mathrm{D}_{4}(\infty)$. These values and Eq. (A4) lead to an equation of the form

$$
\frac{I\left({ }^{5} \mathrm{D}_{3}\right)}{I\left({ }^{5} \mathrm{D}_{4}\right)} \propto \frac{1}{a+b \times p^{E T}} .
$$

${ }^{1}$ For a review article see, e.g., A. J. Kenyon, Prog. Quantum Electron. 26, 225 (2002)

${ }^{2}$ H. J. Lozykowski and W. M. Jadwisienczak, Phys. Status Solidi B 244, 2109 (2007).

${ }^{3}$ W. Carnall, P. Fields, and K. Rajnak, J. Chem. Phys. 49, 4424 (1968); 49, 4443 (1968); 49, 4447 (1968); 49, 4450 (1968).

${ }^{4}$ Phosphor Handbook, 2nd ed., edited by W. M. Yen, S. Shionoya, and H. Yamamoto (CRC Press, Boca Raton, 2007).
${ }^{5}$ J. S. Chang, I. Y. Kim, G. Y. Sung, and J. H. Shin, Opt. Express 19, 8406 (2011).

${ }^{6}$ H. Jeong, Sh. Lee, G. Y. Sung, and J. H. Shin, IEEE Photon. Technol. Lett. 23, 88 (2011).

${ }^{7}$ S. Inaho and T. Hase, "Phosphors for cathode-ray tubes" in Phosphor Handbook, 2nd ed., edited by W. M. Yen, S. Shionoya, and H. Yamamoto (CRC Press, Boca Raton, 2007), Chap. 6.2.

${ }^{8}$ S. B. Aldabergenova, A. Osvet, G. Frank, H. P. Strunk, P. C. Taylor, and A. A. Andreev, J. Non-Cryst. Solids 299-302, 709 (2002).

${ }^{9}$ P. C. Ricci, M. Salis, R. Corpino, C. M. Carbonaro, E. Fortin, and A. Anedda, J. Appl. Phys. 108, 043512 (2010).

${ }^{10}$ Y. Y. Choi, K.-S. Sohn, H. D. Park, and S. Y. Choi, J. Mater. Res. 16, 881 (2001).

${ }^{11}$ F. Benz, J. A. Guerra, Y. Weng, A. R. Zanatta, R. Weingärtner, and H. P. Strunk, J. Lumin. 137, 73 (2013).

${ }^{12}$ W. A. Pisarski, L. Żur, M. Sołtys, and J. Pisarska, J. Appl. Phys. 113, 143504 (2013).

${ }^{13}$ P. C. Ricci, C. M. Carbonaro, R. Corpino, C. Cannas, and M. Salis, J. Phys. Chem. C 115, 16630 (2011).

${ }^{14}$ F. Benz and H. P. Strunk, AIP Adv. 2, 042115 (2012).

${ }^{15}$ D. L. Dexter, J. Chem. Phys. 21, 836 (1953).

${ }^{16}$ T. Förster, Naturwiss. 33, 166 (1946); Ann. Phys. 2, 55 (1948); Discuss. Faraday Soc. 27, 7 (1959).

${ }^{17}$ S. Chandrasekhar, Rev. Mod. Phys. 15, 1 (1943), see page 86.

${ }^{18}$ F. Benz, J. A. Guerra, Y. Weng, R. Weingärtner, and H. P. Strunk, Phys. Status Solidi C 10, 109 (2013).

${ }^{19}$ A. Gonser et al. (to be published); Diploma thesis, University of Stuttgart, Germany (2012).

${ }^{20}$ P. Dorenbos and E. van der Kolk, Opt. Mater. 30, 1052 (2008).

${ }^{21}$ H. J. Lozykowski, W. M. Jadwisienczak, and I. Brown, Appl. Phys. Lett. 76, 861 (2000).

${ }^{22}$ A. Owens and A. Peacock, Nucl. Instrum. Methods A 531, 18 (2004).

${ }^{23}$ C. A. Klein, J. Appl. Phys. 39, 2029 (1968). 